\title{
Semi-Nonparametric Methods for Detecting Latent Non-normality: A Fusion of Latent Trait and Ordered Latent Class Modeling
}

\author{
J. Eric Schmitt \\ Virginia Institute for Psychiatric and Behavioral Genetics \\ Department of Psychiatry \\ Department of Human Genetics \\ Medical College of Virginia \\ Virginia Commonwealth University \\ Paras D. Mehta \\ Department of Psychology \\ University of Houston and \\ Health Research and Policy Centers \\ University of Illinois at Chicago
}

Steven H. Aggen and Thomas S. Kubarych

Virginia Institute for Psychiatric and Behavioral Genetics

Department of Psychiatry

Medical College of Virginia

Virginia Commonwealth University

Michael C. Neale

Virginia Institute for Psychiatric and Behavioral Genetics

Department of Psychiatry

Department of Human Genetics

Medical College of Virginia

Virginia Commonwealth University

Correspondence concerning this article should be addressed to Michael C. Neale, Virginia Institute for Psychiatric and Behavioral Genetics, 800 East Leigh Street, P.O. Box 980003, Richmond, VA 23298-0003. E-mail: neale@vcu.edu 
Ordered latent class analysis (OLCA) can be used to approximate unidimensional latent distributions. The main objective of this study is to evaluate the method of OLCA in detecting non-normality of an unobserved continuous variable (i.e., a common factor) used to explain the covariation between dichotomous item-level responses. Using simulation, we compared a model in which probabilities of class membership were estimated to a restricted submodel in which class memberships were fixed to normal Gauss-Hermite quadrature values. Our results indicate that the likelihood ratio statistic follows a predictable chi-square distribution for a wide range of sample sizes $(N=500-12,000)$ and test instrument characteristics, and has reasonable power to detect non-normality in cases of moderate effect sizes. Furthermore, under situations of large sample sizes, large numbers of items, or centrally located item difficulties, simulations suggest that it may be possible to describe the shape of latent trait distributions. Application to data on the symptoms of major depression, assessed in the National Comorbidity Survey, suggests that the latent trait does not depart from normality in men but does so to a small but significant extent in women.

Most standard factor analyses and structural equation models (SEMs) assume that latent traits follow normal distributions. This assumption follows if one believes that these traits are influenced by a large number of factors, each having a small, equal, and independent effect on the trait. Investigations into the familial resemblance for psychopathology, in particular, often use models based on normally distributed liability that underlies the manifestation of a disease (Kendler, Neale, Kessler, Heath, \& Eaves, 1992). In these models, liability greater than a particular threshold value on a normally distributed latent trait results in pathology (Falconer, 1965). The use of the term liability emphasizes that individual risk factors may not produce an observable effect on the variables of interest, but rather they increase one's risk of contracting disease in concert with other factors. Although its use is perhaps most obvious in genetic epidemiology, the assumption of normality pervades many statistical methods for the analysis of discrete data, perhaps the most notable being tetrachoric and polychoric correlation. It seems reasonable to hypothesize that there is a continuous underlying distribution of liability to psychopathological traits such as depression, because such disorders are complex and liability to them is likely influenced by a multitude of small factors. Thus although psychiatric diagnoses, such as those based on the Diagnostic and Statistical Manual of Mental Disorders (4th ed., DSM-IV; American Psychiatric Association, 1994), are typically dichotomous, the underlying liability to them may not be. Whether this liability is normally distributed is an empirical question, which may be addressed, in part, by the methods we describe in this article.

Investigating the properties of a latent trait distribution can be addressed via latent structure analysis using the patterns of item responses rather than the raw total scores (Lord, 1980). Historically, two general threads of item-level model development can be defined-that of latent trait analyses (LTA) emerging from 
psychometrics and factor analysis (Spearman, 1904) and latent class analyses (LCA) from sociology (Langeheine \& Rost, 1988), although the origin of both can be traced through Lazarsfeld (1950a, 1950b). Whereas LTA typically assumes that the underlying latent variable or variables follow a continuous normal distribution (although other distributions also can be specified), LCA modeling assumes discrete latent variables. In LCA, each individual is assigned a probability of class membership and an item response profile conditional on membership in a particular class. LTA and LCA are not mutually exclusive, however, as an ordered discrete distribution of latent classes can be used to approximate a latent trait (Vermunt, 2004; Vermunt \& Hagenaars, 2004), much as a histogram approximates a continuous distribution. A putative method for testing normality directly follows from this observation, simply by comparing the goodness of fit of two ordered latent class models: the first with probabilities of class membership that are fixed to approximate Gaussian normality (essentially a latent trait model with a discrete distribution instead of a continuous one) and the second relaxing this assumption by specifying weights as free parameters (Vermunt, 2001).

Estimates of quadrature weights can be obtained from several item response theory (IRT) modeling packages, including BILOG-MG (Zimowski, Muraki, Mislevy, \& Bock, 1996) and gllamm (Rabe-Hesketh, Skrondal, \& Pickles, 2004a), although to our knowledge the validity of comparing models with fixed and free weight parameters as a test of latent normality has not yet been established. Here we use a modification of traditional factor analytic methodology to investigate the properties of the latent distribution underlying a set of dichotomous item responses. Normal ogive IRT models and factor models have been formally demonstrated to be equivalent for binary item data, with item difficulty and discrimination reparameterized into thresholds and factor loadings, respectively (Takane \& de Leeuw, 1987). Although in subsequent descriptions we use these terms somewhat interchangeably, parameter values, when given, are scaled in terms of thresholds ( $z$-score units) and standardized factor loadings.

This study employs simulation to demonstrate that the likelihood ratio test used to compare these models is asymptotically distributed as chi-squared. In addition, we explore the statistical power of this method for detecting non-normality in latent trait distributions.

\section{METHOD}

The general statistical approach described next employs a single-factor model implemented in Mx (Neale, Boker, Xie, \& Maes, 2002) that includes free threshold (i.e., difficulty) and factor loading (i.e., discrimination) parameters to describe each item response curve (IRC). The null model differs from traditional LTA in that rather than assuming a normal continuously distributed latent factor, normal- 
ity is approximated using standardized Gauss-Hermite quadratures (Abramozitz \& Stegan, 1972). This approach is equivalent to models in gllamm or Mplus that estimate Gaussian distributions using "ordinary" quadrature (Muthén \& Muthén, 2001; Rabe-Hesketh, Skrondal, \& Pickles, 2004b). Thus, the resultant model conforms mathematically to a (semiparametric) latent class model (Croon, 1990), in that (a) class membership probabilities are given by the quadrature weights located at fixed abscissa values, and (b) item response probabilities are a function of the position of the abscissa on the latent dimension. Conceptually, however, the model is similar to a latent trait model in that the distribution is unidimensional and approximately normally distributed (Figure 1). As we describe in more detail next, the simulations in this study test whether model fit is superior when the central quadrature weights are estimated as free parameters.

Employing IRT models in a traditional factor-analytic framework would pose substantial computational challenges for modest to large numbers of observed items, because likelihood calculations of ordinal data would involve $J$-dimensional integration, where $J$ equals the number of items in the test battery. Marginal maximum likelihood (MML) techniques, however, provide solutions in realistic time

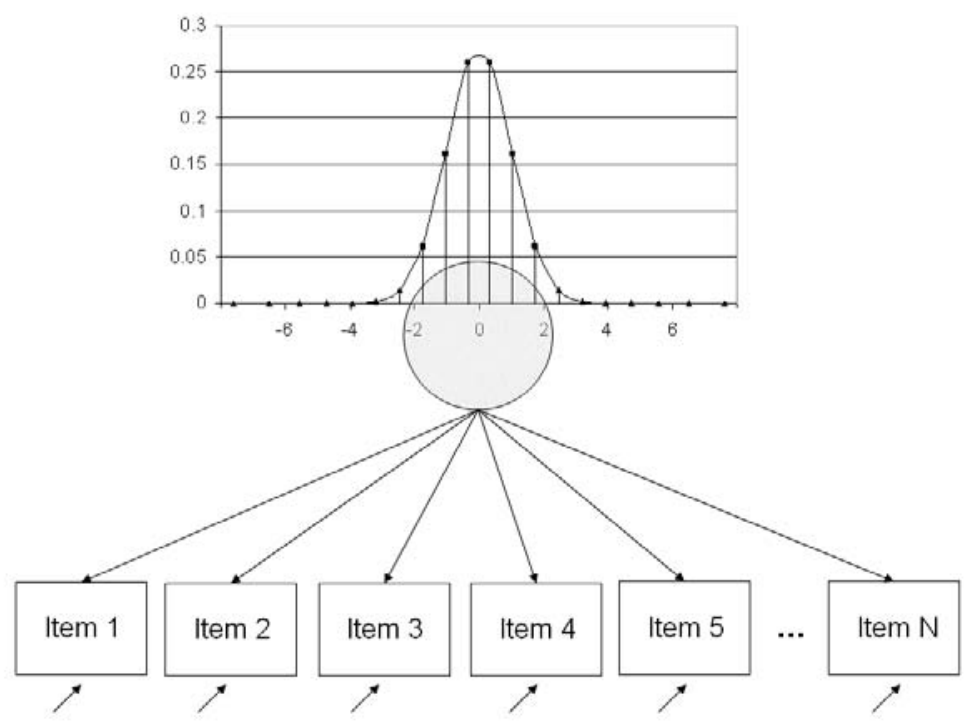

FIGURE 1 Latent trait model schematic, with a 20-point quadrature describing the latent variable as normally distributed. To relax the assumption of normality, the central six quadrature weights (squares) were allowed to fluctuate. 
intervals via integration over (unidimensional) factor space (Bock \& Aitkin, 1981, 1982). Let $X$ represent a unidimensional latent trait that is discretized into 20 quadrature points, with weights $\mathbf{w}$. If $\tilde{V}$ is a matrix of J observed categorical item responses for $N$ individuals, then the likelihood of the observed data can be written as:

$$
P(\tilde{V}=V)=\prod_{n=1}^{N}\left[\sum_{i=1}^{20}\left(P\left(X=w_{i}\right) \prod_{j=1}^{J}\left(P\left(\tilde{V}_{j n}=V_{j n} \mid X=w_{i}, \tilde{L}=L, \tilde{T}=T\right)\right)\right]\right.
$$

where $w_{i}$ indexes the $\mathrm{i}^{\text {th }}$ quadrature weight and $\mathbf{V}_{\text {jn }}$ the $j^{\text {th }}$ item response of subject $n$, and $\mathbf{L}$ and $\mathbf{T}$ are vectors of item factor loading and threshold parameters, respectively. Quadrature was standardized by multiplying Abramowitz and Stegan's (1972) quadrature weights by $\frac{1}{\sqrt{\pi}}$ and the abscissae by $\sqrt{2}$.

Within this framework, a semi-nonparametric latent distribution can be implemented simply by estimating the quadrature weights $w_{i}$ as free parameters, rather than by specifying them as fixed values. The null model is therefore a nested submodel and can be compared directly using likelihood ratio statistics. To this effect, we calculated $\Delta$, twice the difference between the log-likelihood value of the model in which the weights $w_{i}$ were fixed to standardized quadrature values approximating Gaussian normality and the one in which weights were estimated. Three additional constraints were imposed on the model:

$$
\begin{gathered}
\sum_{i=1}^{20} w_{i} a_{i}^{2}=1 \\
\sum_{i=1}^{20} w_{i} a_{i}=0 \\
\sum_{i=1}^{20} w_{i}=1
\end{gathered}
$$

where $w_{i}$ and $a_{i}$ are the weight and abscissa values for quadrature node $i$, respectively. Weights also were bounded within probability space (i.e., $0<w_{i}<1, \forall i$ ). Considered in total, these constraints both standardize the latent trait variable to unit variance and mean of zero (Equations 2 and 3 ) and ensure that the probability space is unity (Equation 4) with no negative probabilities. To minimize the odds of estimated weight parameters encountering the zero boundary (and subsequently influencing the degrees of freedom of hypothesis tests), weights less than .05 in the standardized Gauss-Hermite quadrature were fixed to their corresponding theoret- 
ical weights. The $\Delta$ statistic was expected to follow a chi-squared distribution with degrees of freedom equal to the number of free weights in the semi-nonparametric model (6) minus $3 d f$ to account for the three constraints. For 20-point quadrature, six parameters were freed in the semi-nonparametric model. Thus simulations were expected to follow a chi-squared distribution with $3 d f$, when comparing models with either fixed or free quadrature parameters.

\section{Simulation}

Data sets were simulated using the SAS (SAS Institute, 2000). Under the null hypothesis, the true latent trait is assumed to be normally distributed. Therefore all simulation models assumed a single normally distributed latent trait from which each individual's factor score $\delta_{i}$ was sampled at random. For each item $j$ with factor loading $\lambda_{\mathrm{j}}$, a true score was calculated as $\delta_{i} * \lambda_{j}+\epsilon_{i} \sqrt{\left(1-\lambda_{j}^{2}\right)}$, where $\epsilon_{i}$ is an error term sampled from the standard normal distribution $\mathrm{N}(0,1)$ for each item separately. The item was then scored zero if the individual's true score was less than the item threshold, $T_{j}$, or scored one otherwise. Because of the stochastic element, the simulated items were equivalent to a cumulative normal distribution of item response probabilities for a given factor score (Takane \& de Leeuw, 1987). This procedure was then repeated for all additional items and for all individuals, resulting in a vector pattern of binary item responses for each individual.

We systematically varied sample size; factor loadings; and, to a lesser extent, threshold placement and number of items to investigate test statistic performance under various conditions. In our primary set of simulations, we attempted to approximate the properties of a typical instrument for the assessment of psychopathology (IP), with 9 yes-no items clustered toward the upper range of the latent trait (50\% response probability at $z=-1,0, .25 .50 .751,1.251 .5$, and 1.75). Item loadings were varied from .5 to .9 in increments of .1, in sample sizes of 500; 1,000 ; and then from 2,000 to 12,000 in 2,000 participant increments. In a second set of simulations, we varied the number of items (10 or 15$)$ and the locations (i.e., difficulties-thresholds) of the items in the factor space (equally spaced between -1.6 and $1.6 S D$, centrally clustered $\pm 0.8 S D$, skewed within the $0-1.6 S D$ range, or all set at $z=0$ ). In these simulations, all item factor loadings were .7 and sample size was fixed at 2,000.

To summarize, the simulation procedure consisted of (a) generating data sets in SAS according to a set of specifications described previously (e.g., IP with discriminations of 0.8 and sample size of 6,000 ); (b) simultaneously estimating the thresholds, factor loadings, and the six free weight parameters in Mx using MML and obtaining the $-2 \operatorname{lnL}$ value for each data set; (c) fixing the six free weight parameters in the model to their true normal quadrature values and reestimating the remaining parameters in the model to obtain the $-2 \operatorname{lnL}$ of this submodel; and (d) 
calculating $\Delta$, the difference in -2ln likelihood from the models fit in Steps 2 and 3. This procedure was repeated 1,000 times to obtain a sample distribution of the statistic generated in Step 4.

We then plotted the observed values against the expected distribution $\left(\chi_{3}^{2}\right)$ via chi-squared quantile-quantile (QQ) plots using R (Ihaka \& Gentleman, 1996; R Development Core Team, 2005). QQ plots are scattergrams that plot the expected quantiles from a theoretical distribution (e.g., normal, chi-square) versus the quantiles from the observed data. A line is typically added to the plot to denote the expected $\mathrm{x}$ and $\mathrm{y}$ values if the distribution of the sample statistic conforms to the theoretical distribution. The reader may be most familiar with normal QQ plots, commonly used to identify whether data adheres to normality assumptions prior to the use of parametric statistics. The procedure of simulation, model fit, submodel fit, and generation of QQ plot for 1,000 iterations was repeated for all combinations of simulation parameters.

\section{Power and Situations of Non-Normality}

To characterize the statistical properties of the model under situations in which the null hypothesis was false, we performed a series of simulations with non-normal latent distributions. Given that the number of possible non-normal distributional shapes is infinite, we chose to simulate the most likely distributions (other than Gaussian) to be encountered in reality, namely, mixtures of two normal distributions representing qualitatively distinct populations. These distributions also are interesting because they are intermediate between latent trait (i.e., a single Gaussian distribution) and latent class models (i.e., two or more unrelated distributions with normally distributed errors). In addition, they provide skewed distributions of the latent trait, which are unimodal when the difference $(d)$ between the latent trait means is relatively small, or when the proportion of participants in the larger group $(p)$ was large. We therefore constructed a family of curves by sampling from two distinct standard normal distributions and varying two parameters: (a) $d$, the distance between the means of the two distributions (in $S D$ units), and (b) $p$, the proportion of the sample in the distribution with the lower mean. Simulations were generated for all combinations of $d=0.50,0.75,1.0,1.25$, or 1.5 and $p=.50, .66$, or .90. As an alternative method of generating skewness, we also examined the effect of mixing two normal distributions of equal proportion $(p=.50)$ but unequal variance (lower $S D=1$, upper $S D=0.5$ ) for all values of $d$.

The choice of an optimal item distribution for straightforward comparisons also poses some difficulty. Because the density of true factor scores near a point $x$ on the latent dimension depends on the shape of the latent distribution, the information gleaned from two identically positioned items can differ substantially if the shape of the latent distribution differs. A simple conceptual example of this phenomenon is a test instrument in which items are clustered at the center of a distribution; in the case 
of a Gaussian distribution this test instrument would be highly informative, whereas in a mixture of Gaussian curves this item placement becomes increasingly poor as the distance between the curves increases and approaches bimodality. Thus, to maximize comparability of power calculations between different nonparametric distributions, we placed items at quantiles analogous to the item quantiles in the IP of prior simulations (items at roughly the $16,50,60,69,77,84,89,93$, and 96 th quantiles) rather than at fixed points on the latent distribution based on the z-scores of a parametric distribution (IPQ). We also examined power in the 15-item and polychotomous cases. In the polychotomous case, we added two additional levels to each item in the IPQ (for a total of three thresholds per item), with the upper two thresholds a distance of 0.25 and $0.50 S D$ s above the first item.

To calculate the statistical power of the model, we simulated 100,000 records to minimize sampling error, and then applied the models described previously. For each unique combination of specifications, a $\chi_{3}^{2}$ value testing normality was calculated. By using the "option power" command in Mx, estimates of the sample sizes required to achieve various levels of power for an $\alpha=.05$ are automatically computed, according to the formula:

$$
N^{*}=N^{*} \frac{\lambda_{3}}{X_{3}^{2}}
$$

where $N$ is 100,000 and $\lambda_{3}$ represents the noncentrality parameter for a chi-square test with $3 d f \mathrm{~s}$ and a desired level of power (Neale et al., 2002).

To determine if the maximum likelihood values for the quadrature weights would predict the shape of nonnormal latent distributions (rather than simply determining whether they are nonnormal via hypothesis test), we performed a series of 1,000 simulations for each set of specifications of $d$ and $p$ (e.g., $p=.66$ and $d=$ 1) for a sample size of 6,000 . From these data, we calculated the mean and standard deviation of the six free weight parameters.

\section{Latent Normality Testing for Major Depression}

To provide an example of model performance in actual practice, we analyzed data on major depression (MD) from the National Comorbidity Survey (NCS), acquired by Kessler et al. (1994) as part of a comprehensive examination of mental health, risk factors, and societal effects. This probabilistic survey of 8,098 U.S. adults (4,263 women, 3,835 men) obtained information on numerous psychiatric conditions including affective disturbances, generalized anxiety disorder, phobias, and substance use. Nine dichotomous items based on DSM-III-R criteria for MD were analyzed: (a) depressed mood, (b) loss of interest, (c) weight problems, (d) sleep problems, (e) psychomotor agitation or retardation, (f) tiredness or lack of 
energy, (g) feelings of guilt or worthlessness, (h) trouble concentrating, and (i) thoughts of death or suicide. Factor loadings, thresholds, and weights were estimated for both the semi-nonparametric and Gaussian model; $\Delta$ was calculated to determine if evidence of non-normality could be detected. We then repeated this procedure for men and women separately.

\section{RESULTS}

\section{Simulation}

For most variations of item discrimination and sample size with the IP under the null hypothesis, model comparisons produced chi-square values that closely approximated the expected chi-squared distribution. Figure 2 reports QQ plots for

\section{FACTOR LOADINGS}
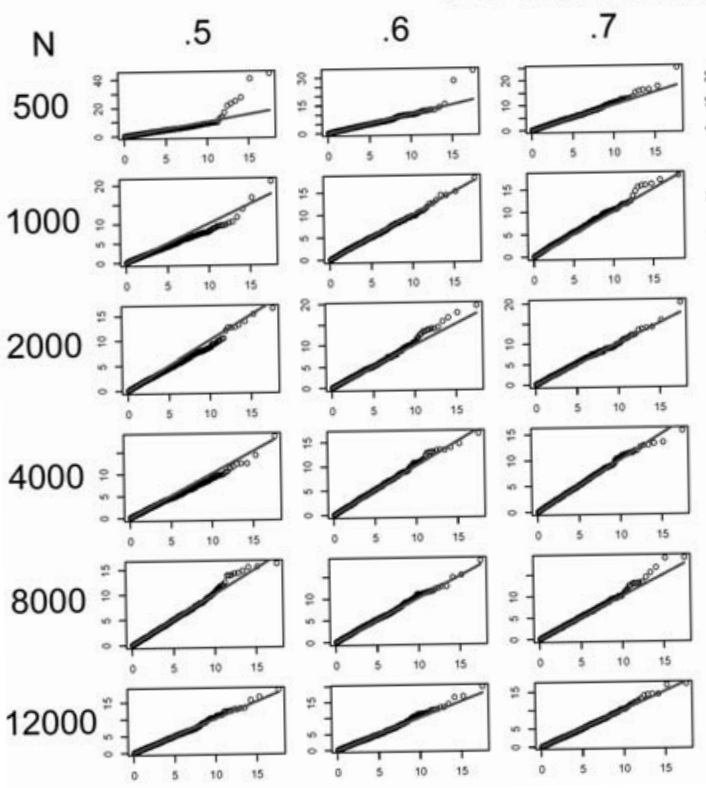

.8
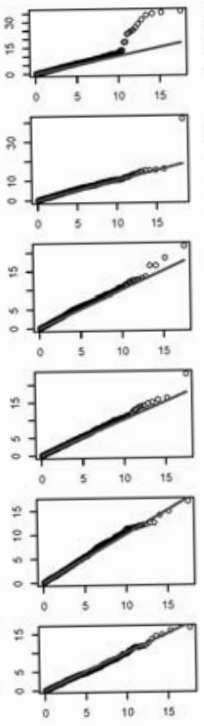

.9
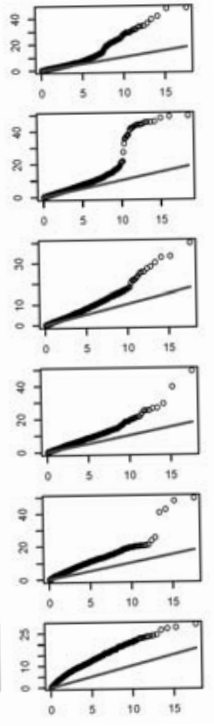

FIGURE 2 QQ plots of 1,000 simulated tests of normality for varying factor loadings and sample sizes. For all plots, items were specified as part of a hypothetical nine-item psychopathological questionnaire, with item placements identical to those shown in Figure 3. A latent trait model with a 20-point quadrature was used to analyze the data. The straight lines represent expectations under a chi-squared distribution with $3 d f$. For simplicity, plots involving sample sizes of 6,000 and 10,000 are not shown, as they had similar patterns to simulations of like sample sizes. 

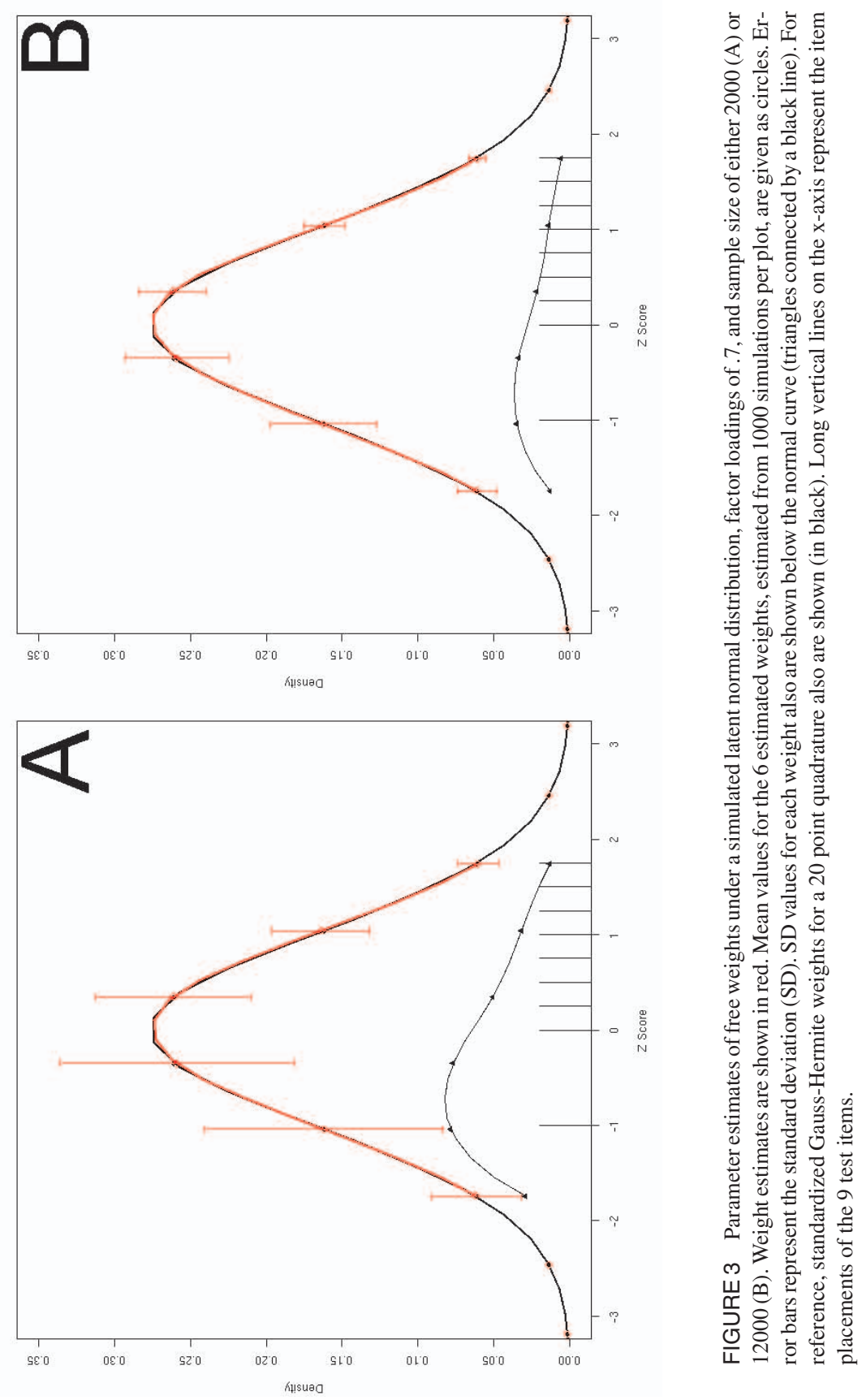
10 ITEM SIMULATIONS
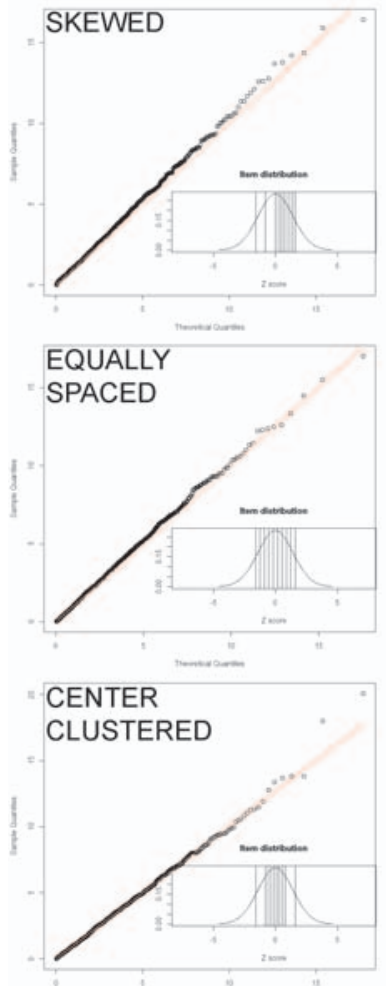

ALL ITEMS

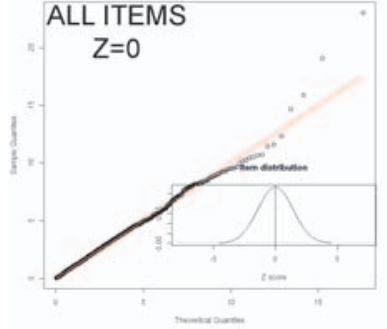

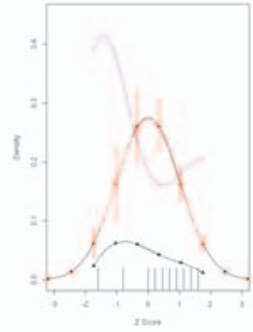
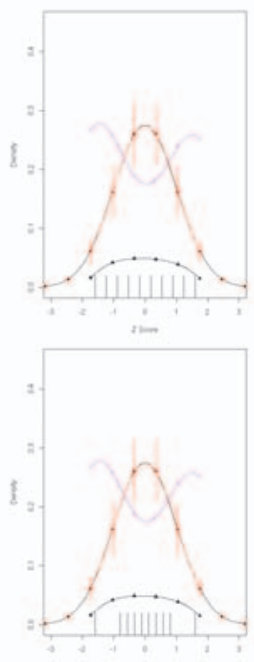

ram

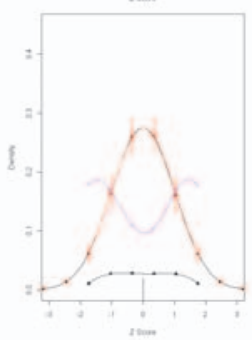

15 ITEM SIMULATIONS
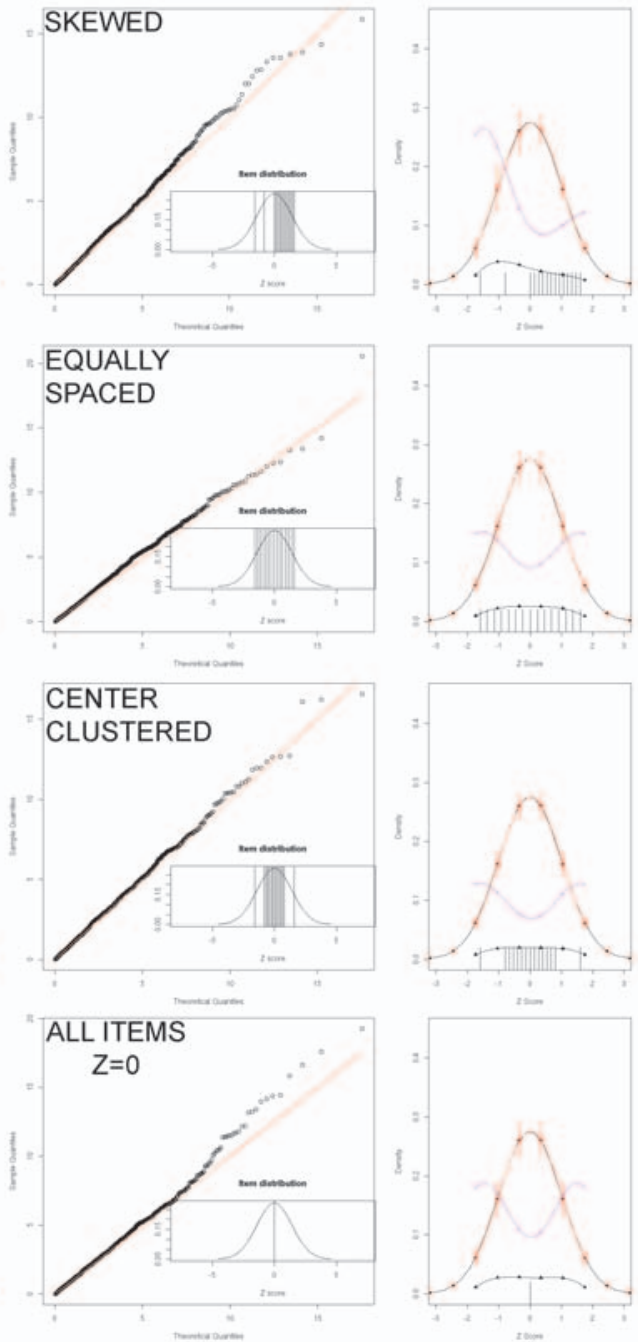

FIGURE 4 Effects of varying item distribution on model performance. Item thresholds for each simulated test battery are displayed in the inset of each QQ plot. Mean values for each quadrature weight are given on profile plots (red open circles), overlaid on the Gauss-Hermite quadrature (black closed circles). The standard deviation of the simulations at each weight are given both as error bars and below the plot (as triangles). The coefficient of variation is shown in purple. Item difficulties are given on the abscissa. The sample size was fixed at 2000 , number of items at 10 or 15 , and item discrimination at .7 for all simulations. 


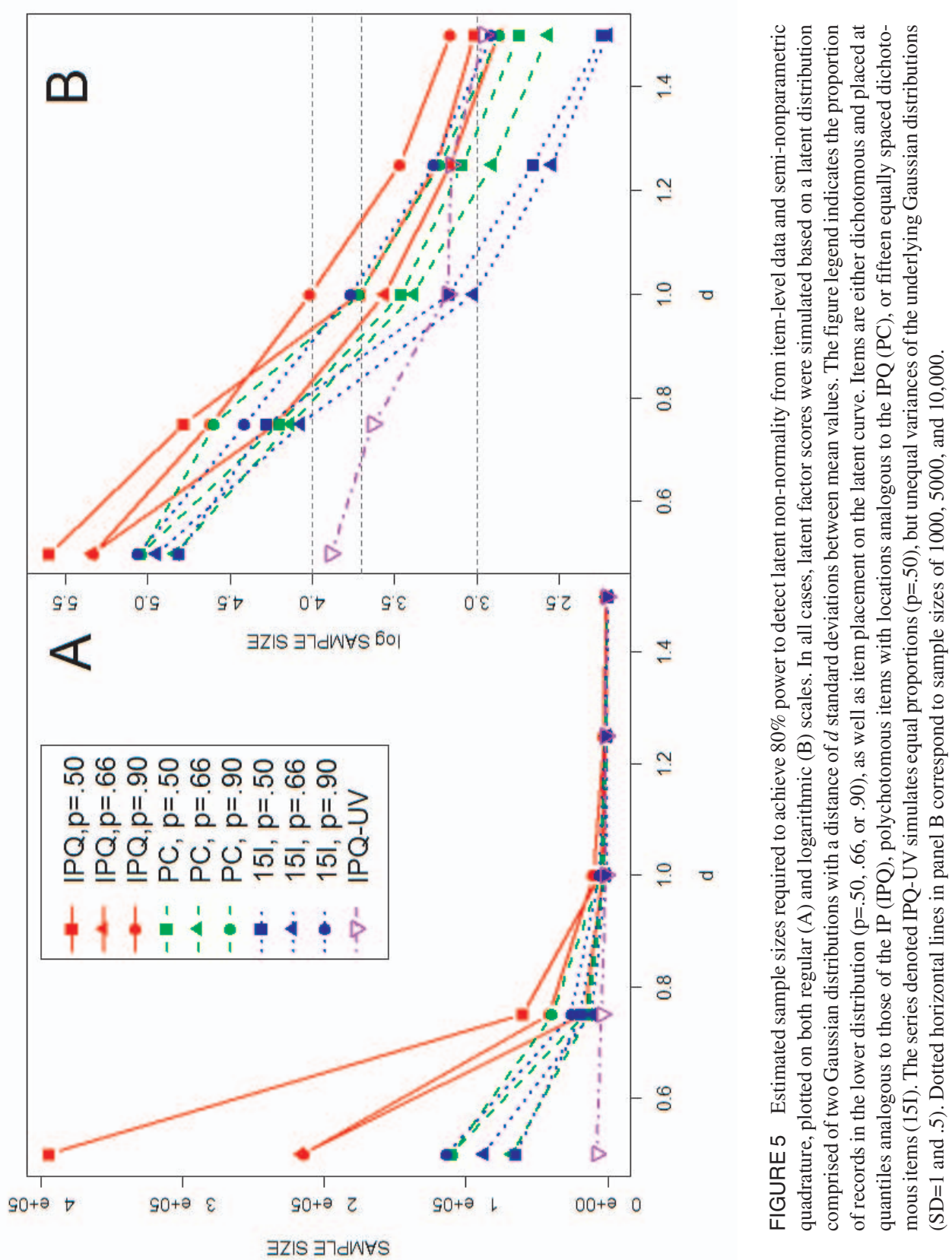




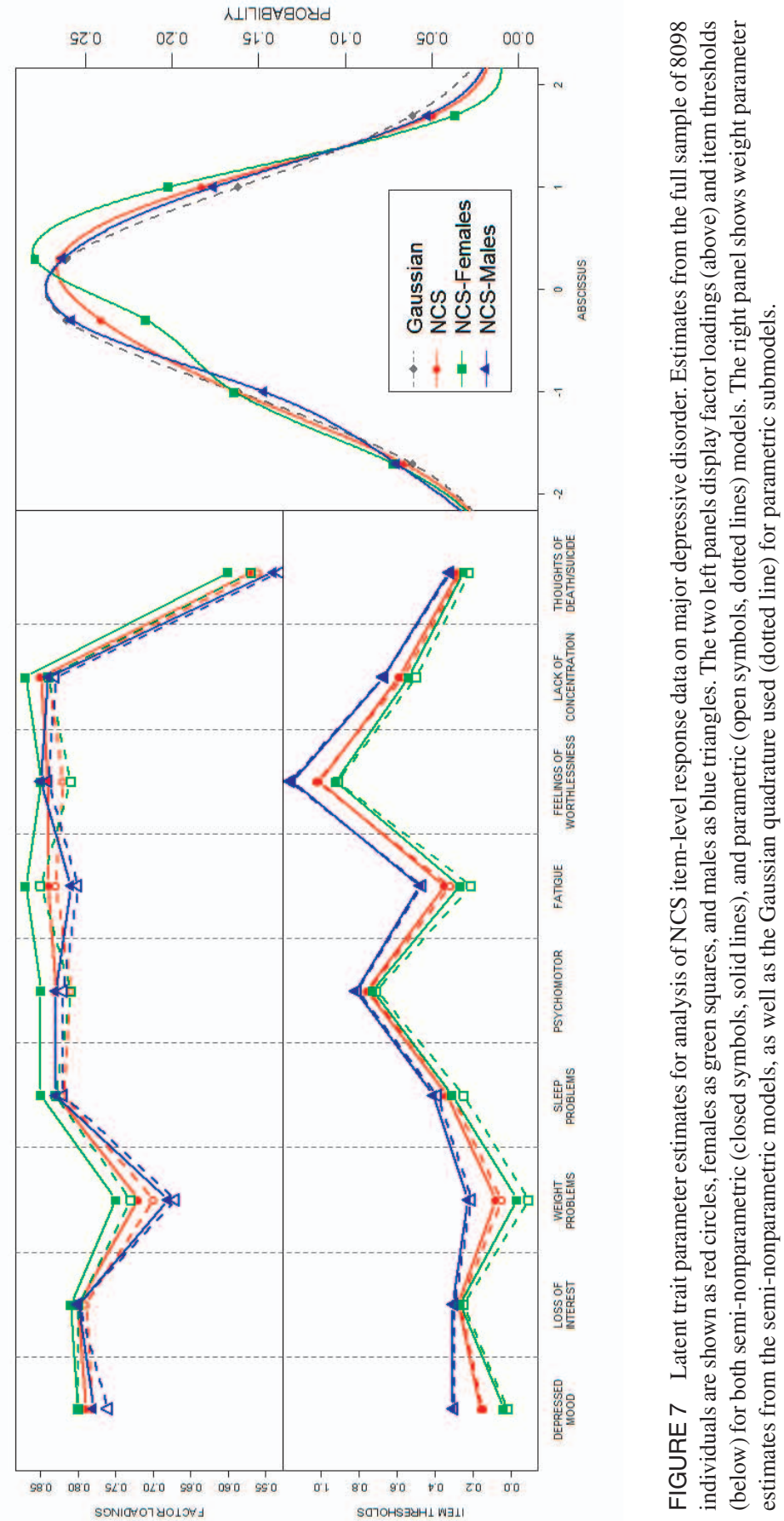


these simulation series. Simulations of item sets with both low discrimination and relatively small sample sizes were somewhat underpowered, but even in the most underpowered case $(N=500$, factor loadings $=.5)$ the observed distribution was close to expectations. In contrast, item sets with factor loadings of .9 produced QQ plots that substantially deviated above expected values, regardless of sample size. This indicates an abrupt change in behavior of the $\Delta$ statistic, as simulations involving factor loadings of .8 tended to follow the expected chi-square distribution closely. Model performance was excellent for factor loadings between .6 and .8 regardless of sample size, and in tests with factor loadings of .5 if the sample size exceeded 6,000 individuals.

Latent contour plots were constructed of estimated weights under the seminonparametric models to determine how well these models predict the "true" normal distributional shape. Figure 3 (see color plate) illustrates typical results observed in most instances. Except for the simulations with factor loadings of .9, the mean weight estimates of models with free weights were indistinguishable from the expected (true population) Gauss-Hermite quadrature weights of the fixed models. The variability of the estimated weight parameters, however, differed substantially according to the sample size, item discrimination, and item threshold location. As expected, simulations with large numbers of participants had small deviations around the true population values, whereas in small sample sizes the variability in weight parameter estimates was quite large. In all cases, the variances of the quadrature weight values were smaller at points on the latent trait near the locations of item difficulties (i.e., where test item information was greatest).

The second series of simulations (see Figure 4 color plate), which varied the number of items and item difficulty parameters, produced results similar to those of the IP series. Regardless of the number of items, the distribution of the test statistic followed the expected chi-squared distribution closely. Similarly, differences in item difficulty locations had little effect on QQ plot values. Mean values for the free weights across simulations agreed with expectations based on the fixed quadrature weights. Variability around the mean weight values was lowest for center-clustered items and tests with more items.

\section{Statistical Power}

Figure 5 (see color plate) provides sample sizes required to obtain $80 \%$ power with $\alpha=.05$. The power of the model was poor when the distance between the two distribution means was small $(d=.50$ or .75$)$ and the shape of the mixture distribution was roughly normal, regardless of $p$ or item placement. As the distance was increased, the sample sizes required to achieve acceptable power decreased dramatically. The addition of more, better placed items also improved the model's sensitivity to non-normality, either by using polychotomous items or by increasing the number of dichotomous items to 15 . 

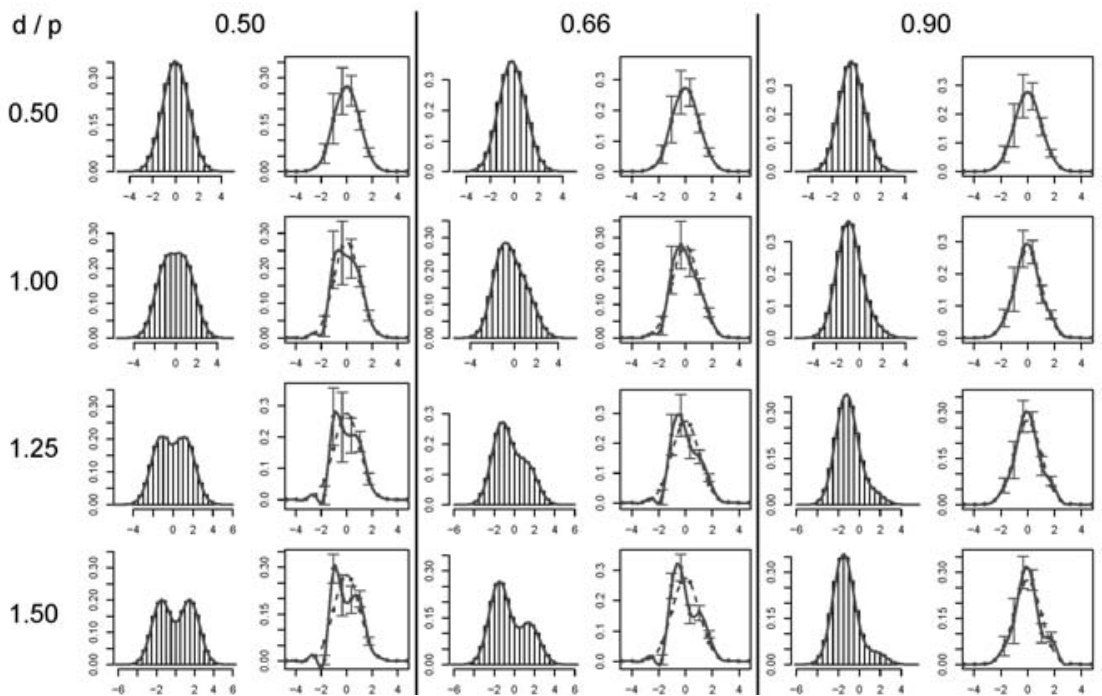

FIGURE 6 Comparisons between true latent distributions and predictions based on analysis of nine dichotomous, right-skewed items. The histograms (with density plot overlayed in red) are calculated from the actual latent factor scores for a random sample of $N=100,000$ used for power calculation. The parameter $d$ represents the distance between two standard normal curves (in $S D$ ) and $p$ is the proportion of factor scores in the left-most distribution. As $d$ increases, the latent distributions increasingly deviate from normality. The plots, shown at right of each corresponding histogram, represent the mean weight estimates for the latent trait derived from item response patterns alone ( $S D$ for each weight as error bars). These plots were based on 1000 simulations, each with $N=6,000$ and factor loadings of .7, and placed according to the IPQ. Expectations under the null hypothesis of normality (as closed gray circles connected by a dotted line) also are shown.

Figure 6 presents histograms of the "true" simulated data beside the model predictions obtained from the analysis of dichotomized item-level information. In most cases, the predictions followed the true distributions. The most striking exception was for a mixture of two equally spaced distributions with large distances between them; in this case the skewed item distribution of the IPQ resulted in some bias in estimating the latent distributional shape.

\section{Application: The Latent Distribution of Major Depression}

The parameter estimates from our analysis of the NCS data are shown in Figure 7 (see color plate). Estimates of both factor loadings and thresholds for item endorsement were approximately equal for the normal and non-normal latent trait models, and for both sexes. Women had slightly higher factor loadings for MD 
items and slightly lower thresholds. Eight of the nine items had strong factor loadings, with suicidal thoughts having by far the weakest relations (loadings $=.54-.60$ for various models and data sets) with the latent factor. All items had less than 50\% endorsement, with the highest threshold for "feelings of worthlessness" at approximately $15 \%$.

Parameter estimates for the six free weights in the semi-nonparametric models also are shown in Figure 7. The full sample had a slight deviation from normality evidenced by the maximum likelihood parameter estimates, which predict a slight rightward skew in the latent variable. These estimates, however, did not significantly deviate from normality $\left(\chi_{3}^{2}=5.59, p=.133\right.$ ) even in this large sample. In men, the maximum likelihood estimates were virtually indistinguishable from expectations under normality assumptions, and the test statistic was correspondingly low $\left(\chi_{3}^{2}=1.20, p=.753\right.$ ). In contrast, when looking at the women alone, the skew was more prominent and was statistically significant $\left(\chi_{3}^{2}=11.56, p=.011\right)$.

\section{DISCUSSION}

These analyses demonstrate that information about latent distributional shapes is retained in categorical item level data and that it can be recovered under certain conditions, even using relatively few dichotomous items. This approach has the potential to inform a broad range of scientific lines of inquiry, from refining definitions of psychopathology and furthering theoretical understanding of psychological traits, to identifying population heterogeneity for research into the genetics of complex disease. At minimum, testing for latent normality prior to latent trait analysis will increase confidence in subsequent parameter estimates and factor scores, which are based on the assumption of a normally distributed latent trait.

Nevertheless, there are some important limitations. Item sets that define a latent trait poorly (i.e., have small factor loadings) will have a slightly higher than expected rate of false negative results, due to lack of statistical power. Yet in reality, item sets with these properties will be quite unusual, as test items are typically chosen to have strong associations to the latent variable of interest. It is more likely that the odd behavior associated with extremely high factor loadings will limit the use of these models in situations in which an excellent test with high discrimination has been developed. A study by van den Oord (2005) also observed this phenomenon in a series of simulations that used Johnson curves to relax latent trait normality assumptions, rather than a quadrature-based approach. Ironically, although items with extremely high factor loadings are superior at discriminating whether individuals are above or below their difficulty than those with lower factor loadings, they provide less information about the rest of the latent distribution, particularly in regions distant from item difficulties. The result is that contingency tables constructed by cross tabulating items from a finite sample of participants 
would have a greater chance of cells with observed frequencies of zero. Thus a lack of broader item information may lead to poor fit under the null hypothesis. This effect also is probably responsible for the extremely high chi-square values seen (albeit rarely) in small samples during our simulation, because cells with zero observed frequencies are more probable with smaller $N$.

A second important limitation is that of power. When the true latent distribution is close in shape to a Gaussian curve (i.e., effect sizes are small), extremely large sample sizes would be required to detect the departure from normality. It should be noted that our simulations with small $d$ are examples in which detecting non-normality is extraordinarily difficult. Even if the latent variable could be observed directly it would be difficult to detect effect sizes equivalent to those of our $d=.5$ or $d$ $=.75$ simulation series.

As expected, power increases dramatically the more the latent trait deviates from the normal distribution; detection of non-normality for extremely non-normal shapes is likely even with sample sizes less than 1,000 . Thus, in situations in which bias is most likely to occur by assuming normality, it is also most likely that the true non-normal shape will be detected. The lack of power may still pose problems for identifying heterogeneity even in large samples; if the deviation from normality is slight, then it is likely not to be detected. However, such minor departures from normality are likely to have little adverse effect on latent trait modeling, and to have little value in describing individual differences in the population. In this article we show that greater statistical power may be obtained in at least two ways. First is to increase the number of items in the test instrument. Doing so may be expected to asymptote in the amount of information about the latent trait; the point at which it will do so is a matter for further research. Furthermore, although developing a large pool item may be straightforward for a trait such arithmetic ability, it may prove difficult or impossible to do for other psychological domains, fears, or phobias. A second approach to improve power is to use polychotomous rather than binary items (van den Oord, 2005). Indeed, a single polychotomous item can be recoded into multiple dichotomous items, assuming an ordered sequence of item responses (Tutz, 1990; van den Berg, Glas, \& Boomsma, 2005), so the analysis of dichotomous items in this study can be considered to be somewhat analogous to an analysis of a smaller number of polychotomous items.

Our results also show that in certain situations quadrature-based latent trait models can be used to recover the actual shape of the latent trait distribution, although at present the approximation may be quite crude. Detection of latent normality can be effective in samples as small as 500 depending on the effect size, but extremely large sample sizes would be required to obtain accurate latent profiles. Alhough the requirement of a large sample will be prohibitive in many studies, application of this approach to large data sets such as the publicly available National Health Interview Survey (NHIS) is feasible. Furthermore, test batteries with many items (or polychotomous data), and those with item difficulties set toward the cen- 
ter of the latent distribution (which allows an item to be informative over a greater proportion of the latent trait density function), will yield more accurate weight parameter estimates. For example, the 2,000-participant simulation with 10 items and $z=0$ had comparable weight variances to the IP simulations with $N=12,000$ but skewed item thresholds. Thus, test batteries with favorable properties, either by chance or by design, may be able to estimate the shape of the latent trait distribution even in modest sized samples.

The concept of placing latent classes along the continuum of latent traits is an old one, and is described by Lazarsfeld and Henry (1968) in their seminal text as a "located class" model. These authors noted that OLCA models are particularly prone to failures of the assumption of local independence due to class inhomogeneity; this point may be partially responsible for the relative dearth of research on ordered latent classes despite an explosion of interest in both LTA and LCA. In our models, the use of a relatively high-resolution quadrature may ameliorate potential problems due to small deviations from class homogeneity. Despite the potential limitations of OLCA, substantive contributions have been made (Clogg, 1988; Croon, 1990; Heinen, 1993; Langeheine, 1988; van Onna, 2004; Vermunt, 2001), although with different methods than those described here. For example, several other methods for describing latent non-normality have been developed (de Leeuw \& Verhelst, 1986; Follman, 1988; Magder \& Zeger, 1996; van den Oord, 2005) using MML and a variety of approaches for modeling non-Gaussian latent distributions, each with their strengths and weaknesses. Quadrature-based methods generally involve more free parameters than methods that use functional forms (e.g., Johnson curves or multiple Gaussian curves) and may be more prone to sample fluctuations (van den Oord, 2005). The use of quadrature, however, may also provide more accurate estimates of complex latent distributional shapes, as well as more reliable performance with dichotomous data (van den Oord, 2005).

One alternative approach to testing for normality, due to Pearson (1900), uses the traditional chi-square test. This method compares the observed frequencies of scores in a finite number of categories with the frequencies that would be expected if the data were normally distributed. This classical test should not be confused with the approach described in this article. Here we compare, via likelihood-ratio test, a model with estimated quadrature weights to one with weights fixed a priori to Gaussian quadrature values. Under certain regularity conditions, this test is asymptotically distributed as chi-squared with degrees of freedom equal to the number of free parameters used to estimate the weights. Another approach might be to compute factor scores based on the item response patterns of the test participants, and to compare these factor scores with the normal distribution, using either the classical or a more modern test. However, this approach would inevitably perform badly when there are floor or ceiling effects, such that obtaining either the maximum or the minimum score on the test is not uncommon. All factor scores from such participants would be the same, so the factor score distribution would depart 
from normality. This type of problem is likely to be especially acute when the number of items is small.

Although preliminary, we believe that this study demonstrates the potential utility of the analysis of the shape of latent distributions. The first empirical application of the model provides some evidence to this effect. Using NCS data, these analyses suggest that the latent factor underlying major depression is normally distributed when considering men and women together or men only, but deviates from normality somewhat in women. At present, any comments on the etiology of a female rightward skew would be speculative, although it is not difficult to imagine several mechanisms that would produce this effect. Regardless, in the case of MD, these models demonstrate that parameter estimates of factor loadings and thresholds differ little between parametric and semi-nonparametric models.

These analyses also suggest several directions for future research. First, it would be useful to understand the behavior of these models under situations of very high thresholds (e.g., $z>2$ ), which would increase the utility for researchers investigating relatively rare conditions such as schizophrenia, phobias, personality disorders, or conduct disorder. Second, characterization of individual factor scores under situations of non-normality may ultimately enable classification of subpopulations with different etiologies, which could in turn be useful in subsequent genetic or psychometric analysis. Third, employing adaptive rather than static quadrature may result in an increased flexibility of the models and improve precision of weight estimation; therefore implementation of adaptive quadrature in this context is a worthwhile endeavor. Fourth, the methods described above are easily extended to modeling multidimensional latent distributions, and further simulation study should be performed to demonstrate its effectiveness in this respect. Multidimensional models would be useful for characterizing either the resemblance between relatives, or the comorbidity between traits. Last, it would be useful to determine the performance of models with varying item discriminations, as well as in the face of deviations from the fundamental assumptions of IRT, such as unidimensionality and local independence.

\section{ACKNOWLEDGMENT}

This research was supported by National Institutes of Health grants MH-65322, MH-20030, and DA-18673.

\section{REFERENCES}

Abramowitz, M., \& Stegan, I. E. (1972). Handbook of mathematical functions with formulas, graphs, and mathematical tables (10th ed.). Washington, DC: U.S. Government Printing Office. 
American Psychiatric Association. (1994). Diagnostic and statistical manual of mental disorders (4th ed.). Washington, DC: Author.

Bock, R., \& Aitkin, M. (1981). Marginal maximum likelihood estimation of item parameters: Application of an EM algorithm. Psychometrika, 46, 443-459.

Bock, R., \& Aitkin, M. (1982). Errata: "Marginal maximum likelihood estimation of item parameters: Application of an EM algorithm." Psychometrika, 47, 369.

Clogg, C. C. (1988). Latent class models for measuring. In R. Langeheine \& J. Rost (Eds.), Latent trait and latent class models (pp. 173-205). New York: Plenum.

Croon, M. A. (1990). Latent class analysis with ordered latent classes. British Journal of Mathematical and Statistical Psychology, 43, 171-192.

de Leeuw, J., \& Verhelst, N. (1986). Maximum-likelihood-estimation in generalized Rasch models. Journal of Educational Statistics, 11, 183-196.

Falconer, D. S. (1965). The inheritance of liability to certain Diseases, estimated from the incidence among relatives. Annals of Human Genetics, 51-76.

Follman, D. (1988). Consistent estimation in the Rasch model based on nonparametric margins. Psychometrika, 53, 553-562.

Heinen, T. (1993). Discrete latent class models. Tilburg, The Netherlands: Tilburg University Press.

Ihaka, R., \& Gentleman, R. (1996). R: A language for data analysis and graphics. Journal of Computational and Graphical Statistics, 5, 299-314.

Kendler, K. S., Neale, M. C., Kessler, R. C., Heath, A. C., \& Eaves, L. J. (1992). A population-based twin study of major depression in women: The impact of varying definitions of illness. Archives of General Psychiatry, 49, 257-266.

Kessler, R. C., McGonagle, K. A., Zhao, S., Nelson, C. B., Hughes, M., Eshleman, S., et al. (1994). Lifetime and 12-month prevalence of DSM-III-R psychiatric disorders in the United States: Results from the National Comorbidity Survey. Archives of General Psychiatry, 51, 8-19.

Langeheine, R. (1988). New developments in latent class theory. In R. Langeheine \& J. Rost (Eds.), Latent trait and latent class models (pp. 77-108). New York: Plenum.

Langeheine, R., \& Rost, J. (1988). Introduction and overview. In R. Langeheine \& J. Rost (Eds.), Latent trait and latent class models (pp. 1-7). New York: Plenum.

Lazarsfeld, P. F. (1950a). The interpretation and computation of some latent structures. In S. A. Stouffer (Ed.), Measurement and prediction (pp. 413-472). Princeton, NJ: Princeton University Press.

Lazarsfeld, P. F. (1950b). The logical and mathematical foundation of latent structure analysis. In S. A. Stouffer (Ed.), Measurement and prediction (pp. 362-412). Princeton, NJ: Princeton University Press.

Lazarsfeld, P. F., \& Henry, N. W. (1968). Latent structure analysis. Boston: Houghton Mifflin.

Lord, F. M. (1980). Applications of item response theory to practical testing problems. Hillsdale, NJ: Lawrence Erlbaum Associates, Inc.

Magder, L. S., \& Zeger, S. L. (1996). A smooth nonparametric estimate of a mixing distribution using mixtures of Gaussians. Journal of the American Statistical Association, 91, 1141-1151.

Muthén, L. K., \& Muthén, B. O. (2001). Mplus user's guide. Los Angeles: Muthén \& Muthén.

Neale, M. C., Boker, S. M., Xie, G., \& Maes, H. H. (2002). Mx: Statistical modeling (5th ed.) [Computer software]. Richmond: Department of Psychiatry, Medical College of Virginia, Virginia Commonwealth University.

Pearson, K. (1900). On the criterion that a given system of deviations from the probable in the case of a correlated system of variables is such that it can be reasonably supposed to have arisen from random sampling. Philosophical Magazine, 50, 157-175.

$\mathrm{R}$ Development Core Team. (2005). R: A language and environment for statistical computing. The $\mathrm{R}$ Foundation for Statistical Computing, Vienna, Austria.

Rabe-Hesketh, S., Skrondal, A., \& Pickles, A. (2004a). Generalized multilevel structural equation modeling. Psychometrika, 69, 167-190. 
Rabe-Hesketh, S., Skrondal, A., \& Pickles, A. (2004b). GLLAMM manual. (Working Paper 160). UC Berkeley Division of Biostatistics Working Paper Series, Berkeley, CA. Available from http://www.bepress.com/ucbbiostat/paper160

SAS Institute. (2000). SAS/STAT User's Guide (Version 8) [Computer software]. Cary, NC: Author.

Takane, Y., \& de Leeuw, J. (1987). On the relationship between item response theory and factor analysis of discretized variables. Psychometrika, 52, 393-408.

Tutz, G. (1990). Sequential item response models with an ordered response. British Journal of Mathematical and Statistical Psychology, 43, 39-55.

van den Berg, S. M., Glas, C. A. W., \& Boomsma, D. I. (2005). Variance decomposition using an IRT measurement model. Manuscript submitted for publication.

van den Oord, E. J. (2005). Estimating Johnson curve population distributions in MULTILOG. Applied Psychological Measurement, 29, 45-64.

van Onna, M. J. H. (2004). Ordered latent class models in nonparametric item response theory. Groningen, The Netherlands: Groningen University Library.

Vermunt, J. K. (2001). The use of restricted latent class models for defining and testing nonparametric and parametric item response theory models. Applied Psychological Measurement, 25, 283-294.

Vermunt, J. K. (2004). An EM algorithm for the estimation of parametric and nonparametric hierarchical nonlinear models. Statistica Neerlandica, 58, 220-233.

Vermunt, J. K., \& Hagenaars, J. A. (2004). Ordinal longitudinal data analysis. In R. C. Hauspie, N. Cameron, \& L. Molinari (Eds.), Methods in human growth research (pp. 374-393). Cambridge, UK: Cambridge University Press.

Zimowski, M. F., Muraki, E., Mislevy, R. J., \& Bock, R. D. (1996). BILOG-MG: Multiple-group IRT analysis and test maintenance for binary items [Computer software]. Chicago: Scientific Software International. 